\title{
EBSD Analysis of the Submicron Width Fibber Shaped Grain Copper Fabricated by Drawing
}

\author{
Masafumi Matsushita $^{1^{*}}$, Tomoya Kuji ${ }^{2}$, Hiromitsu Kuroda ${ }^{2}$, Seigi Aoyama ${ }^{2}$, Hiroaki Ohfuji ${ }^{3}$ \\ ${ }^{1}$ Department of Mechanical Engineering, Ehime University, Bunkyocho, Matsuyama, Japan; ${ }^{2}$ Metallic Materials Research Depart- \\ ment, Hitachi Cable. Ltd., Kawajiri, Hitachi, Japan; ${ }^{3}$ Geodynamics Research Center, Ehime University, Bunkyocho, Matsuyama, \\ Japan. \\ Email: *matsushita@eng.ehime-u.ac.jp
}

Received February $16^{\text {th }}, 2011$; revised March $21^{\text {st }}, 2011$; accepted May $5^{\text {th }}, 2011$.

\begin{abstract}
Several drawing processes of $3 \mathrm{~N}$ pure copper $(\mathrm{Cu})$ with ultra high reduction in area have been performed, and the texture has been observed using electron back scattered diffraction. It is well known that the texture of drawn Cu is closely related to its mechanical properties; in particular submicron scale width fibber shaped grain is interesting. Previously, it was reported that drawing $3 \mathrm{~N} \mathrm{Cu}$ with around $95 \%$ reduction in area changes its mechanical properties. In the present experiment, grains have been changed from round to fiber-shaped, and subsequently, submicron-width fiber grains were generated with a $94.546 \%$ reduction in area. However, above $94.546 \%$ reduction in area, dynamic recrystallization occurred and then, prevented the grains to be finer. Further recrystallized grains influence on the macroscopic mechanical properties of $\mathrm{Cu}$ wire. Furthermore, the distribution of the misorientation angle at grain boundaries increased with an increase in the degree of reduction in area; in particular, the distribution drastically increased between $94.546 \%$ and $99.997 \%$. Further, the frequency of coincidence of site lattice boundaries increased. The abovementioned variations in texture are closely related to mechanical properties.
\end{abstract}

Keywords: Drawing, Grain Boundary, EBSD, Cu, Fibber Shape Grain

\section{Introduction}

The mechanical properties of metals and alloys are greatly influenced by their grain size, shape, and misorientation angle at grain boundaries (GBs). It is well known that the effect of grain size on tensile strength follows the Hall-Petch equation,

$$
\sigma_{Y}=\sigma_{0}+k d^{-1 / 2}
$$

where $\sigma_{Y}$ denotes yield strength, $d$ denotes grain diameter, and $\sigma_{0}$ and $k$ are material constants [1].

Furthermore, regarding the fiber grain shape, the tensile strength of the grain in the oblong direction increases with a decrease in the width of the fiber, obeying the following Hall-Petch type equation,

$$
\sigma_{Y}=\sigma_{0}+k W^{-1 / 2},
$$

where $W$ is the fiber grain width [2].

However, the Hall-Petch equation is not valid in nanometer scale grains $[3,4]$. The material having macroscopic grain, the yield strength increases obeying Hall-Petch equation. On the other hand, grain being submicron size, yield strength is obeying Hall-Petch equation, inter-granular fracture and local shear deformation increase [3]. Further decrease of grain size, yield strength is not obeying Hall-Petch equation and many interesting phenomenon appear, for example, nanocrystalline $\mathrm{Cu}$ of the diameter $20 \mathrm{~nm}$, which is made by electro deposition and rolling, shows super plasticity [5]. However the relation between fibber width of fibber shape grain and mechanical behavior does not clear. Further the behavior of dynamic strength, specifically fatigue strength, is not clear.

In recent times, bulk metals having nanometer scale grains, the so-called bulk nano-metals, are considered as potential next-generation structured materials [6]. Accordingly, several methods for the fabrication of bulk nano-metals have been proposed, although, most of these methods are not suitable for fabrication on an industrial scale [7-10]. The drawing process is one of the severe plastic deformation processes and can be used for industrial fabrication. In particular, the drawing of $\mathrm{Cu}$ is a common method for fabricating many industrial products such as trolley wire and magnet wire. Meanwhile, the 
demands for $\mathrm{Cu}$ wire with specific mechanical properties have increased with the recent developments of the electric car, industrial robots, and so on.

Severe drawing deformation will make submicron width fibber shape grain. Previous studies reported the relation between the mechanical properties and the reduction in area by drawing. Hardness and tensile strength increase in direct proportion to the reduction in area just below $95 \%$ (hereafter we call this range as stage 1). At around $95 \%$ reduction, the tensile strength and hardness show a drastic increase (hereafter we call this range as stage 2). However furthermore increase in the area reduction, the tensile strength and hardness become constant (hereafter we call this range as stage 3). The twist fatigue strength test was performed for an effective length of $65 \mathrm{~mm}$, a diameter of $2.8 \mathrm{~mm}$, and at the rate of 1000 times $/ \mathrm{min}$. Twist fatigue strength also increases in direct proportion to the reduction in area just below $95 \%$, namely in stage 1 . And then at around $95 \%$ reduction, namely in stage 2, twist fatigue strength shows a drastic increase. However, as the area reduces further, namely in stage 3 , twist fatigue strength decreases while the tensile strength and hardness is constant $[11,12]$. This discontinuous behavior of hardness, tensile and twist fatigue strengths depended on reduction area and mismatch of the variation among hardness, tensile and twist fatigue strengths are so interesting. It is considered that abovementioned variations of mechanical properties depending on the reduction in area are closely relation to the texture variations, but the textures, such as grain size, grain boundary characters have not been clear.

With the recent developments in electron back scattered diffraction (EBSD), we can determine the misorientation angle information of GBs and the crystal orientation of the local area, as the result, grain boundary engineering have been developed [13]. The textures of drawn metal with ultra high reduction in area have not been investigated by EBSD thus far. In this study, we have performed EBSD measurements of the longitudinal dimensions of drawn $\mathrm{Cu}$ wires corresponding to the abovementioned three stages in order to demonstrate the textures of drawn metal, such as the grain size, shape, preferred orientation of the grains and GB misorientation angle. Furthermore, the relationships among these features and the mechanical properties of the material have been discussed.

\section{Experimental Method}

\subsection{Sample Preparation}

The samples were fabricated by the drawing of $99.9 \%$ pure $\mathrm{Cu}$ with various reductions in area, as listed in Table 1 (hereafter, these sample are referred to by the
Table 1. A list of reduction in area of each sample.

\begin{tabular}{cc}
\hline Sample No. & Reduction in area (\%) \\
\hline 1 & 73.450 \\
2 & 94.546 \\
3 & 99.997 \\
\hline
\end{tabular}

assigned numbers). The fabrication process involved performing the drawing process several times. After the copper was drawn once, the area was reduced by $15 \%$. For the sake of convenience in the EBSD experiments, the final diameters of all the samples were kept at $42 \mu \mathrm{m}$. The samples were embedded in resin, and then their longitudinal cross sections were polished using diamond powder and colloidal silica with diameters of $1 \mu \mathrm{m}, 50$ and $20 \mathrm{~nm}$.

\subsection{Electron Backscattered Diffraction Measurement Method}

The longitudinal cross sections of each sample were then observed through EBSD. The measurement area was positioned halfway from the central axis, as shown in Figure 1, since the texture of the drawn $\mathrm{Cu}$ wire samples was symmetric around the central axis. The measurement step of the EBSD was $100 \mathrm{~nm}$, and the accelerated voltage of the electron beam was $15 \mathrm{kV}$. Channel 5 (HKL Technology, Co.) was used for analysis. The minimum misorientation angle defined in the present experiment was $1^{\circ}$.

In this paper, we have classified the GB into the following three types: 1) coincidence site lattice (CSL) boundaries, having higher geometric coordination; 2) low-angle grain boundaries (LGBs), which have a misorientation angle of $<15^{\circ}$; and 3) high-angle grain boundaries (HGBs), which have a misorientation angle of $>15^{\circ}$ but are not CSL grain boundaries. HGBs do not have specific rules and include some atomic voids $[14,15]$.

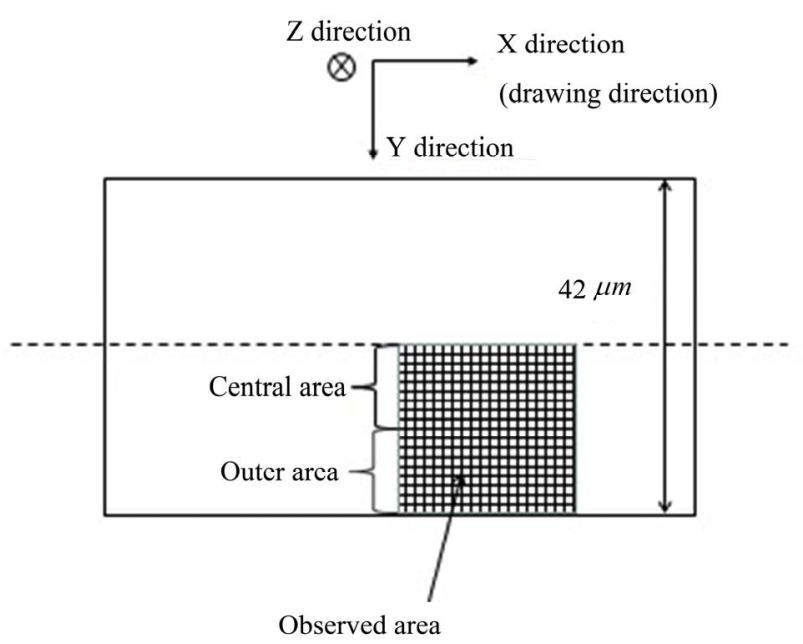

Figure 1. Observed longitudinal area of $\mathrm{Cu}$ wire. 


\section{Results and Discussions}

\subsection{Grain shape and Grain Boundary Characteristics}

Grain boundary maps of each sample listed in Table $\mathbf{1}$ are shown in Figure 2. The black, red, and thin gray lines are HGBs, $\Sigma 3$ boundaries, and LGBs, respectively. The $\Sigma 3$ boundary has the highest geometric coordination, namely, twin boundary.

The grain shape changes from a round to a fiber shape when the area is reduced, as shown in Figures 2(a) and (b). The fiber width becomes several hundred $\mathrm{nm}$ and the aspect ratio is larger than 5, as shown in Figure 2(b). Deformation twinning by room temperature plastic deformation has not been reported previously. However, present EBSD measurement results clearly show the existence of deformation twin. It is considered that this elongation of the nano-fiber grain along the drawing direction is mainly responsible in increasing the tensile and twists fatigue strengths.

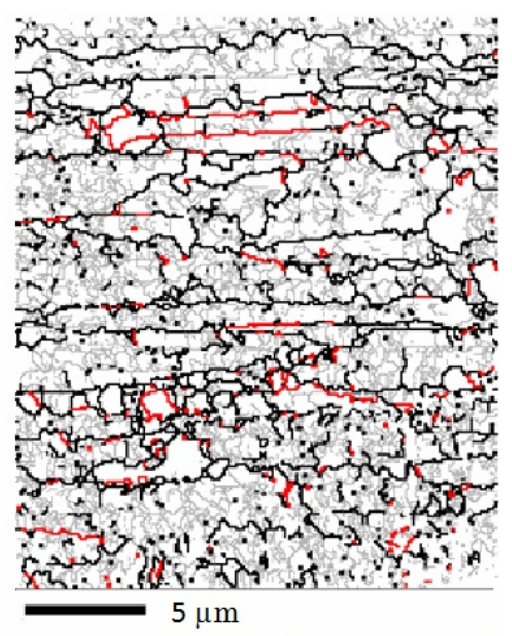

(a)

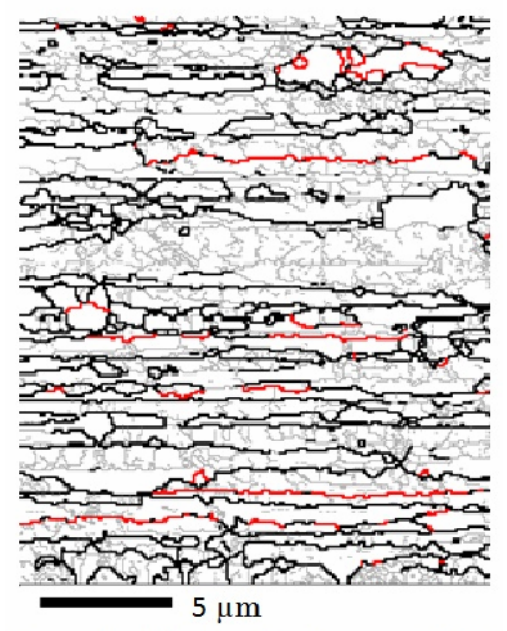

(b)

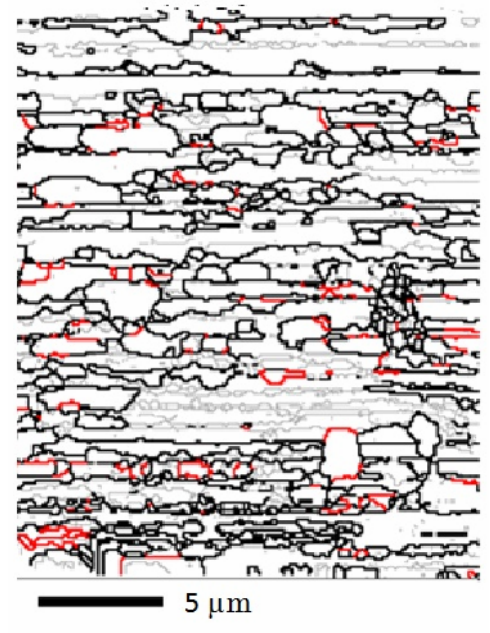

(c)

Figure 2. Grain boundary maps of samples. (a), (b) and (c) are Sample 1, 2 and 3, respectively. Thin gray lines show LGBs. Red and black lines show $\Sigma 3$ CSL boundaries and HGBs, respectively. (a) Sample 1 (reduction in area $73.450 \%$ ); (b) Sample 2 (reduction in area 94.546\%); (c) Sample 3 (reduction in area $99.997 \%$ ).

With further reduction in area, round grains appear and some of them are oblong to the central axis, as shown in Figure 2(c). These grains are considered as recrystallized grains. Main reason of recrystallization is the strain energy generated by deformation. Moreover, there is possibility that solute atoms of $\mathrm{Cu}$ are concentrated in HGBs by severe plastic deformation, and then a part of the grain becomes the starting point for dynamic recrystallization. These dynamic recrystallizations prohibit the grain becoming finer.

The frequency of CSL boundaries is shown in Figure 3. CSL boundaries increase with an increase in the reduction in area, particularly in sample 3. However, the relative frequency for the misorientation angle of the CSL boundary is much lower than $\mathrm{Cu}$ of similar area recrystallized grain obtained equal channel angular extrusion [10]. The relative frequencies for the misorientation angle of each sample are shown in Figure 4. The relative frequency of HGBs drastically increases as the percentage reduction in area grows from $94.546 \%$ to $99.9997 \%$. GBs with misorientation angles of $<5^{\circ}$ decrease drastically. The recrystallized grains effect on the macroscopic mechanical behavior of materials. Furthermore, an increase in the relative frequency of HGBs causes inter-granular fracture by impact [16]. Recrystallized grain and the increase of HGBs influence tensile and twist fatigue strengths. In particular, twist fatigue strengths is changed between stage 2 and 3, which correspond sample 2 and 3 . It means intergranular fracture occurs at HGBs is one of reasons of this change. Further, 


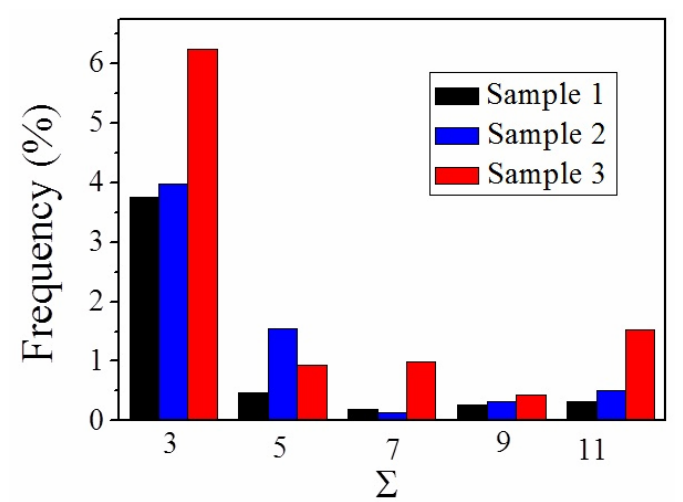

Figure 3. Frequency of CSL boundaries at each sample.

grain boundary sliding would occur in stage 3 as same in case of non-fibber submicron grain [4].

\subsection{Preferred Orientations}

Inverse pole figures (IPFs) of the outer and central area of each sample are shown in Figure 5.

In sample 1 , very weak $<100>+<111>$ double orientation textures are confirmed in the drawing direction, namely, the $\mathrm{X}$ direction; these textures are stronger in the outer area. In the $\mathrm{Y}$ and $\mathrm{Z}$ directions, a clear orientation direction cannot be seen; that is, the direction of the radius has a random orientation.

In sample 2, the clear preferred orientation is confirmed in the $\mathrm{X}$ direction. In the central area, a $<100>$ axis orientation is seen. Furthermore, a $<100>$ weak orientation is confirmed in the $\mathrm{Y}$ and $\mathrm{Z}$ directions. These results indicate that a cube texture was formed. In the outer area, $<100\rangle+<111\rangle$ double axis orientations are seen in the $\mathrm{X}$ direction. The $<100>$ orientation is weak compared to the center area. Further rotation from $<100>$ to $<101>$ occurs in the $\mathrm{Z}$ direction. Considering these results, a (100) [100] cube orientation is found to collapse by shear stress.

IPFs of sample 3 are shown in Figure 5. Very strong orientations are confirmed in the $\mathrm{X}$ direction. In the outer area, $\mathrm{a}<111>$ axis orientation is clearly confirmed. In the central area, a $<100>$ rich $<100>+<111>$ double axis orientation is confirmed. The radius direction is in both $\mathrm{Y}$ and $\mathrm{Z}$ directions, and a weak $<101>$ axis orientation is clearly seen in both the outer and center areas. Considering these results, a $<111>$ axis orientation is generated, and the cube orientation shown in sample 2 disappears. A strong $<111>$ axis orientation in the $\mathrm{X}$ direction would affect the mechanical properties since the slip direction is vertical to the drawing direction.

The preferred orientation of recrystallized $\mathrm{Cu}$ wire by annealing, i.e., before drawing the sample, has a $<001>$ axis orientation in the $\mathrm{X}$ direction and $\mathrm{a}<101>$ axis ori-

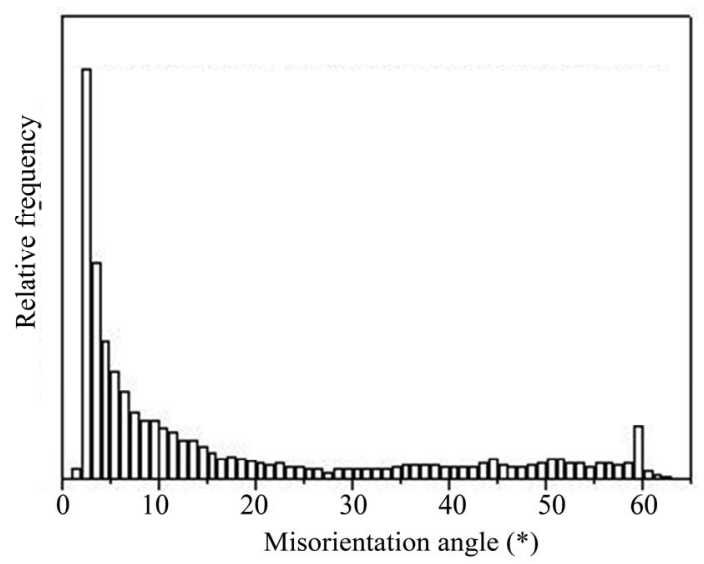

(a)

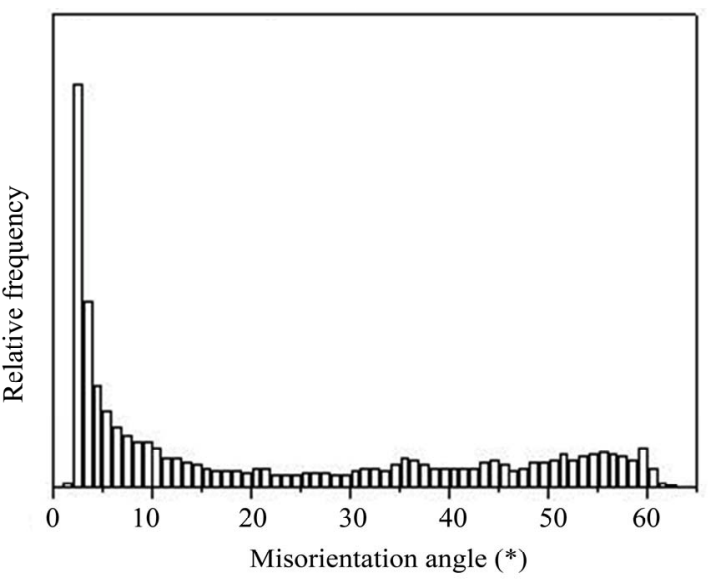

(b)

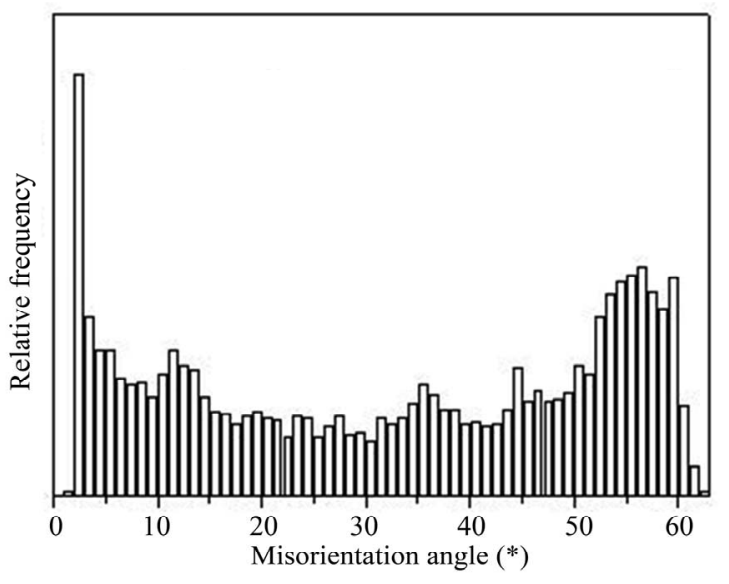

(c)

Figure 4. Relative frequencies for the misorientation angle of each sample. (a), (b) and (c) are Sample 1, 2 and 3, respectively. (a) Sample 1 (reduction in area $\mathbf{7 3 . 4 5 0 \% ) ; ~ ( b ) ~}$ Sample 2 (reduction in area 94.546\%); (c) Sample 3 (reduction in area $99.997 \%$ ).

entation in the $\mathrm{Y}$ and $\mathrm{Z}$ directions.

Brown discussed the relationship between the orientation of the fiber texture rotation and the stacking fault 

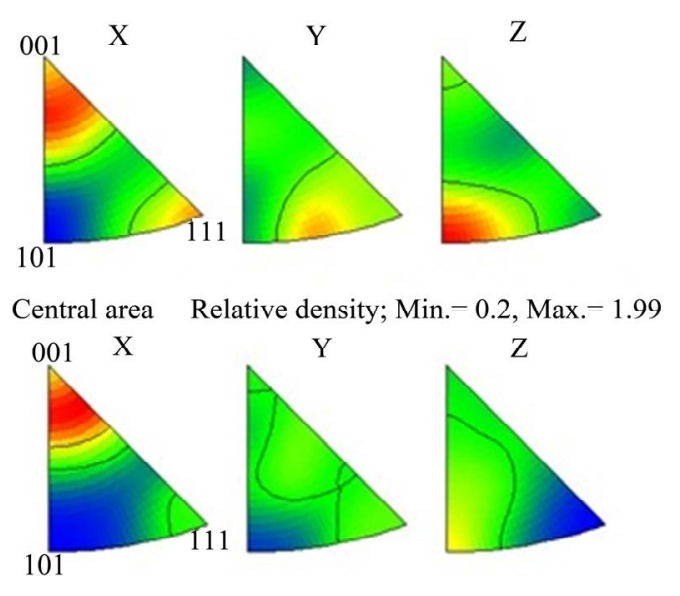

Out area Relative density; Min. $=0.05$, Max. $=2.87$

(a)

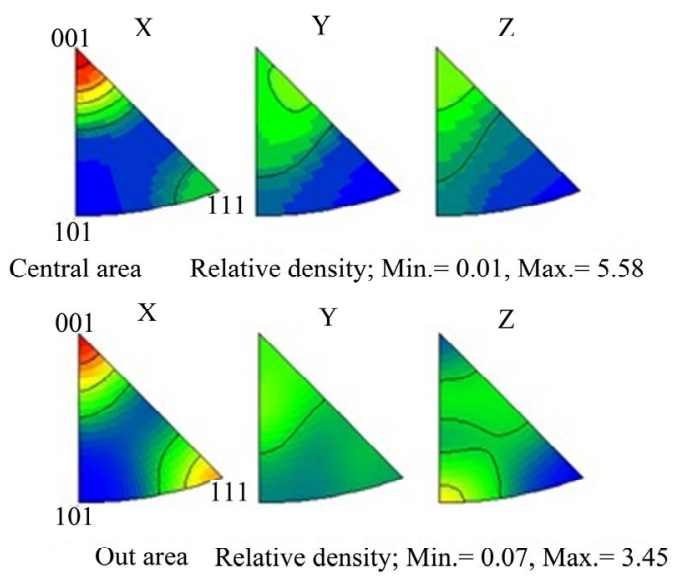

(b)
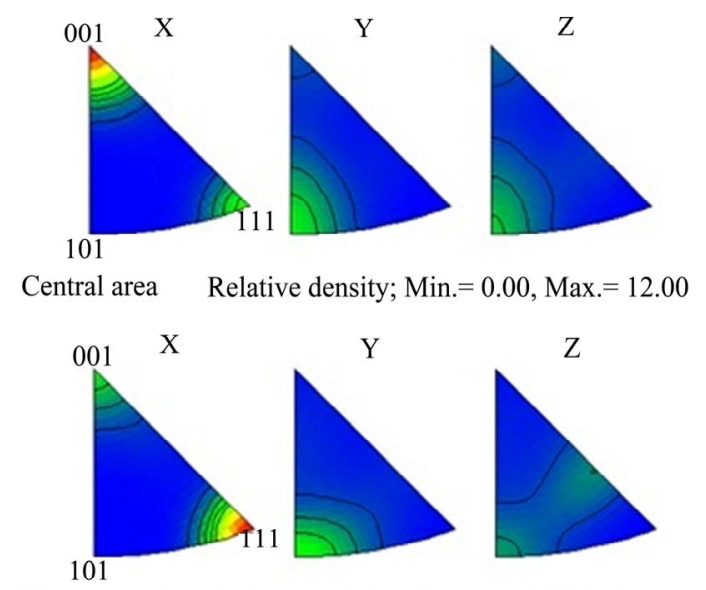

Out area Relative density; Min. $=0.01$, Max. $=11.53$

(c)

Figure 5. Inverse pole figures of the outer and central areas of each sample. (a), (b) and (c) are Sample 1, 2 and 3, respectively. (a) Sample 1 (reduction in area $73.450 \%$ ); (b) Sample 2 (reduction in area 94.546\%); (c) Sample 3 (reduction in area $99.997 \%$ ). energy (SFE) [17]. According to his theory, a high SFE metal causes cross slip more easily than a low SFE metal, and subsequently, the cross slip leads to the rotation of the $<100>$ axis orientation to $<111>$. The SFE of Al is higher than that of $\mathrm{Cu}$. The present results compared to past experimental results for $\mathrm{Al}$ [18], obtained through $\mathrm{X}$-ray diffraction, show that the $<100>$ axis orientation of $\mathrm{Al}$ rotates to $<111>$ in lower than that of $\mathrm{Cu}$. This result corresponds to ref. [17], and cross slips are considered to be the origin of this change.

During the drawing process, a two-axis stress system, corresponding to compression and tensile stress, is added to the central area. On the other hand, in the outer area, a strong shear stress parallel to the drawing dice is added to the two-axis stress of the central area. Inakazu has analyzed the orientation change caused by the abovementioned stress systems and proved the stability of the $<100>$ and $<111>$ anises [19]. However, in [19], orientation change from $<001>$ to $<112>$ and then $<111>$ is predicted with an increase in the reduction in area. Furthermore, these changes are confirmed in $\mathrm{Al}$ experimentally. In the present experiment for $\mathrm{Cu}$, the data almost corresponds to the results of a previous experiment for $\mathrm{Al}$; however, the $\mathrm{X}$-axis orientation does not change via $<112>$.

\section{Conclusions}

Grains of $\mathrm{Cu}$ were changed to submicron-width fiber grains with ultra high reduction in area. However, when the degree of reduction in area was increased for 94.546 and $99.997 \%$, dynamic recrystallization occurred. Recrystallizations prevent the grain to be finer and influence on the macroscopic mechanical properties, which mentioned in ref. [11,12].

The distributions of the relative frequency of HGBs increase with an increase in the degree of reduction in area; this increase in relative frequency distribution is drastic between 94.546 and $99.997 \%$. Furthermore, the frequency of CSL boundaries increases. These changes in textures would cause a discontinuous change in mechanical properties reported in ref. [11] and [12]. Considering from the present grain and grain boundaries character changes, intergranular fracture and grain boundary slip would cause the remarked change of twist fatigue fracture.

Preferred orientations of drawing and radius directions change to the $<111>$ axis. These changes occur for higher reductions in area for $\mathrm{Cu}$ rather than $\mathrm{Al}$, because the SFE of $\mathrm{Al}$ is smaller than that of $\mathrm{Cu}$.

\section{REFERENCES}

[1] E. O. Hall, "Yield Point Phenomena in Metals and Al- 
loys," Plenum Press, New York, 1970.

[2] Y. Sakai, "Strength of Heavily Cold Worked Two-PhaseCopper Alloy," Materia Japan, Vol. 36, 1997, pp. 692696.

[3] T. G. Nieh and J. Wadsworth, "Hall-Petch Relation in Nanocrystalline Solid," Scripta Metallurgica et Materialia, Vol. 25, No. 4, 1991, pp. 955-958. doi:10.1016/0956-716X(91)90256-Z

[4] P. G. Sanders, J. A. Eastman and J. R. Weertman, "Elastic and Tensile Behavior of Nanocrystalline Copper and Palladium," Acta Materialia, Vol. 45, No. 10, 1997, pp. 4019-4025. doi:10.1016/S1359-6454(97)00092-X

[5] L. Lu, M. L. Sui and K. Lu, "Superplastic Extensibility of Nanocrystalline Copper at Room Temperature," Science, Vol. 287, No. 5457, 2000, pp. 1463-1466. doi:10.1126/science.287.5457.1463

[6] L. L. Shaw, "Processing Nanostructured Materials: An Overview," JOM Journal of the Minerals, Metals and Materials Society, Vol. 52, No. 12, 2000, pp. 41-45. doi:10.1007/s11837-000-0068-2

[7] Y. Saito, H. Utsunomiya, N. Tsuji and T. Sakai, "Novel ultra-High Straining Process for Bulk Materials - Development of the Accumulative Roll-Bonding (ARB) Process," Acta Materialia, Vol. 47, No. 2, 1999, pp. 579-583. doi:10.1016/S1359-6454(98)00365-6

[8] Z. Horita, D. J. Smith, M. Furukawa, M. Nemoto, R. Z. Valiv and T. G. Langdon, "An Investigation of Grain Boundaries in Submicrometer-Grained Al-Mg Solid Solution Alloys Using High-Resolution Electron Microscope," Journal of Materials Research, Vol. 11, No. 8, 1996, pp. 1880-1890. doi:10.1557/JMR.1996.0239

[9] A. Belyakov, W. Gao, H. Miura and T. Sakai, "StrainInduced Grain Evolution in Polycrystalline Copper during Warm Deformation," Metallurgical and Materials Transactions A, Vol. 29, No. 12, 1998, pp. 2957-2965. doi:10.1007/s11661-998-0203-1

[10] G. Wang, S. D. Wu, L. Zuo, C. Esling, Z. G. Wang and G. Y. Li, "Microstructure, texture, Grain Boundaries in Re- crystallization Regions in Pure Cu ECAE Samples," Materials Science and Engineering A, Vol. 346, No. 1-2, 2003, pp. 83-90. doi:10.1016/S0921-5093(02)00521-X

[11] H. Yamamoto and N. Inakazu, "On the Relation between Drawing Ratio and Mechanical Properties for Copper Wire," Journal of the Japan Copper and Brass Research Association, Vol. 11, 1974, pp. 133-141.

[12] N. Inakazu and H. Kawakami, "Drawing," In: H. Tanaka, Ed., The Japan Society of Technology of Plasticity, Corona Publishing Co. Ltd., Tokyo, 1994, pp. 80-86.

[13] V. Randle, "Grain Boundary Engineering: An Overview after 25 Years," Materials Science and Technology, Vol. 36, No. 3, 2010, pp. 253-261. doi:10.1179/026708309X12601952777747

[14] L. N. Brewer, M. A. Othon, L. M. Young and T. M. Angeliu, "Misorientation mapping for Visualization of Plastic Deformation via Electron Back-Scattered Diffraction," Microscopy and Microanalysis, Vol. 12, No. 1, 2006, pp. 85-91. doi:10.1017/S1431927606060120

[15] M. A. Arafin and J. A. Szpunar, "Modeling of Grain Boundary Character Reconstruction and Predicting Intergranular Fracture Susceptibility of Texture and Random Polycrystalline," Computional Matterials Science, Vol 50, No. 2, 2010, pp. 656-665. doi:10.1016/j.commatsci.2010.09.031

[16] T. Watanabe, "The Impact of Grain Boundary Character Distribution on Fracture in Polycrystals," Material Science and Engineering A, Vol. 176, No. 1-2, 1994, pp. 39-49. doi:10.1016/0921-5093(94)90957-1

[17] N. Brown, "The Dependence of Wire Texture in FCC Metals on Stacking Fault Energy," Transactions of Metallization Society AIME, Vol. 221, 1969, pp. 236-238.

[18] N. Inakazu and H. Yamamoto, "A Study on the Process of Fiber Texture Formation in Aluminium," The Japan Institution of Metals, Vol. 37, 1973, pp. 1224-1229.

[19] N. Inakazu, "Metal Drawing," Kindai Hensyu Publishing Co. Ltd., Tokyo, 1985, p. 157 